\title{
ION BACK-BOMBARDMENT OF GAAS PHOTOCATHODES INSIDE DC HIGH VOLTAGE ELECTRON GUNS*
}

\author{
J. Grames ${ }^{\#}$, P. Adderley, J. Brittian, D. Charles, J. Clark, J. Hansknecht, M. Poelker, M. Stutzman, \\ K. Surles-Law, Thomas Jefferson National Accelerator Facility, Newport News, VA 23606, U.S.A.
}

\begin{abstract}
DC high voltage GaAs photoguns are key components at accelerator facilities worldwide. New experiments and new accelerator facilities demand improved performance from these guns, in particular higher current operation and longer photocathode operating lifetime. This conference submission explores bulk GaAs photocathode lifetime as a function of beam current, active photocathode area, laser spot size and the vacuum of the gun and beam line. Lifetime measurements were made at $100 \mu \mathrm{A}$, a beam current relevant for accelerators like CEBAF, and at beam currents of $1 \mathrm{~mA}$ and $5 \mathrm{~mA}$, a regime that is interesting for high current Free Electron Laser (FEL) and Energy Recovery Linac (ERL) operation.
\end{abstract}

\section{INTRODUCTION}

The quantum efficiency (QE) of GaAs photocathodes within state-of-the-art DC high voltage photoemission electron guns degrades primarily as a result of ion backbombardment [1,2] where residual gas between the cathode-anode gap is ionized by the extracted electron beam and back-accelerated toward the photocathode. Ions with sufficient kinetic energy damage the photocathode surface or possibly sputter away the chemicals used to create the negative electron affinity (NEA) condition. The rate of $\mathrm{QE}$ degradation depends strongly on the rate of ion production which is a function of the vacuum condition within the gun. Good base vacuum within the gun chamber and transport beam line is essential for obtaining long photocathode lifetime and it is critical that vacuum remain good while delivering beam.

During the early years of the polarized electron beam program at CEBAF, it was recognized that some of the beam from the photocathode originated from locations not intentionally illuminated by laser light. These "extra" electrons were produced by stray light from the laser table caused by diffraction at laser apertures or from spurious laser beams originating from numerous reflective surfaces. In addition, spontaneous emission within the photocathode material produces light that can be reabsorbed by the photocathode, either directly or as a result of internal reflections within the reflective electropolished gun vacuum chamber. Photoemission from the center of the photocathode was properly managed by the electron-optic transport system and delivered to the accelerator but electrons emitted from the edge of the photocathode traveled extreme trajectories and would eventually strike the vacuum chamber walls, liberating gas via electron stimulated desorption. When this happened, gun vacuum degraded and photocathode lifetime was unacceptably short. To solve this problem, the edge of the photocathode surface was anodized in an electrolytic bath [3]. The center of the photocathode still provided high QE, but the anodized edge no longer produced photoemission. This successfully eliminated unwanted photoemission from the cathode edge and improvement in photocathode lifetime at CEBAF was immediately obvious. Other accelerators have since recognized the utility of limiting photocathode active area as a means to prolong photocathode lifetime $[4,5]$.

\section{EXPERIMENT}

The experimental setup, shown in Fig. 1, was arranged to investigate the dependence of photocathode lifetime on beam intensity, photocathode active area, laser spot size and vacuum pressure by systematically varying the

- $\quad$ active area: no mask (13 $\mathrm{mm})$ or masked $(5 \mathrm{~mm})$,

- laser spot size: small (0.5 mm) or large (1.2 mm),

- beam current $(100 \mu \mathrm{A}, 1 \mathrm{~mA}$ or $5 \mathrm{~mA})$.

Details of the setup follow in this section.

The electron gun consists of a load lock chamber to introduce and heat a bulk GaAs sample, a preparation chamber with a mask to limit the active area when cesium and nitrogen trifluoride are applied, and a $100 \mathrm{kV}$ high voltage chamber to accelerate the photoemitted electrons. Prior to changing either the active area or laser spot size the sample was moved to the load lock chamber for an extended heating $(9-15 \mathrm{hr}$ at $575 \mathrm{C}$ ). This process allowed the QE to be largely restored, allowing the one sample to be used repeatedly. The cathode QE, measured 12 hours after activation, was routinely $12-19 \%$ at $532 \mathrm{~nm}$.

A Coherent Verdi-10 laser was selected for the sufficiently large QE expected at its wavelength $(532 \mathrm{~nm})$. The spot size at the cathode was set by a combination of a telescope beam expander and, just prior to the beam line vacuum window, a focusing lens to locate the laser spot on the photocathode. The laser intensity profile was

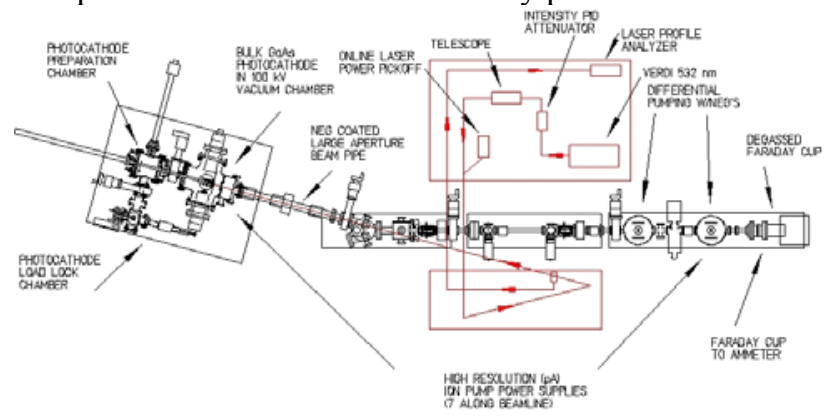

Figure 1: (Color) The $100 \mathrm{kV}$ DC electron gun, beam line and laser system used for this experiment is shown.

\footnotetext{
*Work supported by the U.S. DOE, contract DE-AC05-84ER401050 "grames@jlab.org
} 
measured by diverting the laser beam, after the focusing lens, to a laser profile analyzer located at the photocathode distance. The laser power and wavelength were measured by reflecting a small fraction of the incident beam with a glass slide to an optical meter. The laser intensity was remotely controlled by a combination of a variable attenuator and neutral density filters, and also measured in-line by an optical power meter.

The electron gun is located at $15^{\circ}$ with respect to the remainder of the beam line to allow the laser to reach the photocathode at normal incidence. A series of air-core steering coils and five solenoid magnets $(f \sim 50 \mathrm{~cm})$ transport the beam 5.5 meters to a Faraday cup. Viewers assist to reproducibly setup the beam through the center of the solenoids and beam pipe. Magnetic shielding was added to dampen ambient AC magnetic fields.

The base vacuum in the gun chamber is $1.6 \times 10^{-11}$ Torr using an extractor gauge corrected for $\mathrm{H}_{2}$ (dominant species $>99.9 \%$ ) and the beam line vacuum does not exceed $10^{-10}$ Torr. This was achieved by an extended bake-out at $250 \mathrm{C}$, the use of non-evaporable getters (NEG) and ion pumps. High resolution $(<50 \mathrm{pA})$ ion pump power supplies provide for continuous relative monitoring of the extremely low pressures and are sensitive monitors for beam loss via desorbed gas. To improve vacuum isolation of the photocathode from the dumped beam, the Faraday cup was degassed at $450 \mathrm{C}$ and a second differential pump was added. The vacuum isolation of the photocathode dramatically improved $(>20 x)$ and beam induced pressure rise is now dominated by electron loss along the beam line.

Prior to each run the vacuum pressure and initial $\mathrm{QE}$ were measured. The beam originated from within the central $2 \mathrm{~mm}$ diameter of the photocathode, typically

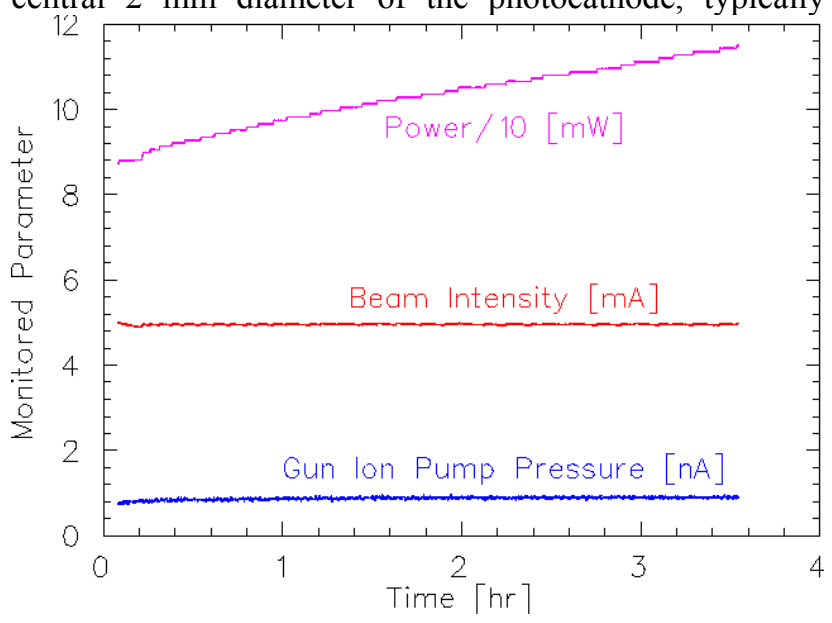

Figure 2: (Color) A $5 \mathrm{~mA}$ run; the laser power is automatically increased to maintain constant beam current that, in turn, induces an increased pressure.

the same physical spot. The beam orbit was first set at low current and the current was then raised to the desired value by adjusting the laser attenuator. While maintaining the beam current constant using a PID loop to lock the photocurrent, laser power and vacuum pressure were recorded, e.g., in Fig. 2.

\section{RESULTS}

For each new configuration of either active area or laser spot size, three runs of $100 \mu \mathrm{A}(9 \mathrm{C}), 1 \mathrm{~mA}(29 \mathrm{C})$ and 5 $\mathrm{mA}(72 \mathrm{C})$ were conducted to determine photocathode lifetime, defined as the charge extracted for the QE to fall by $1 / \mathrm{e}$ of the initial value.

The lifetime for all runs is reported in Fig. 3 as a function of the average vacuum condition. The unmasked lifetimes correlated to the degraded vacuum in the gun

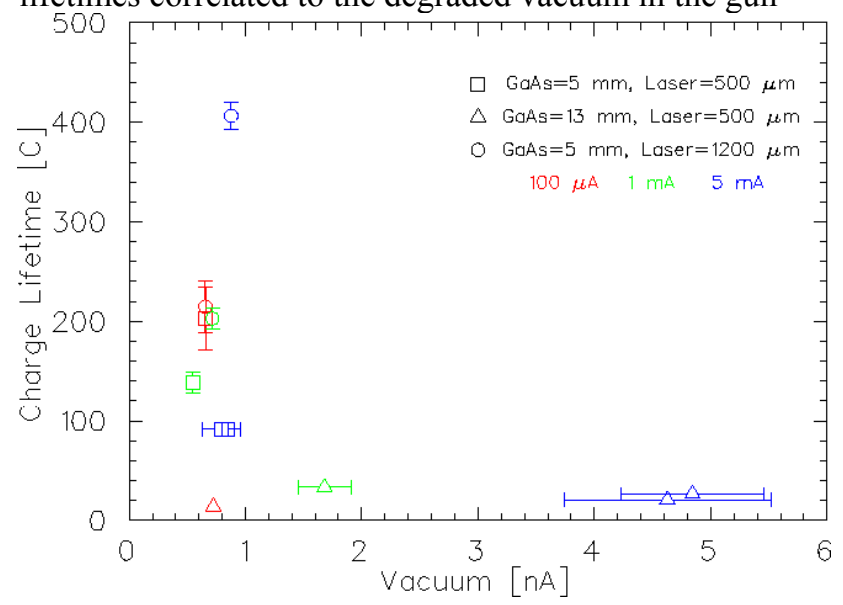

Figure 3: (Color) Photocathode lifetime versus vacuum pressure during the run (base vacuum $\sim 0.5 \mathrm{nA}$ ).

have a marked contrast with the improved masked lifetimes and marginal increase over the initial vacuum, regardless of the laser spot size and beam intensity. Limiting the cases to only masked photocathodes one may expect the longer lifetimes to occur at larger laser spot size because, for a given current, ion damage is distributed over a larger area. These results, with masked active area $(5 \mathrm{~mm})$ are shown in Fig. 4. The anticipated scaling of lifetime with laser spot size indeed occurs at 5 $\mathrm{mA}$, however, as the current is reduced the enhancement

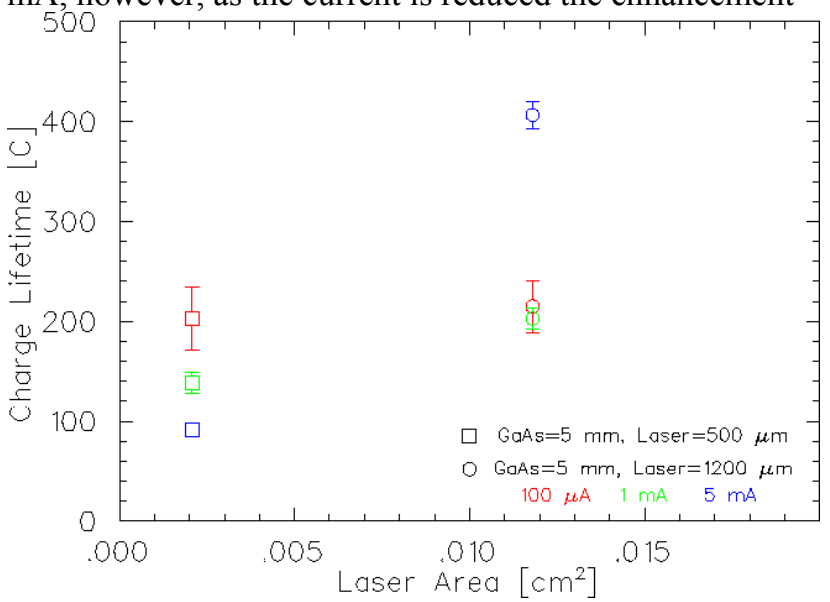

Figure 4: (Color) Photocathode lifetime versus laser spot size with the active area diameter fixed at $5 \mathrm{~mm}$.

with spot size, unexpectedly, is also reduced. Because the absolute vacuum pressure is similar between all runs (0.6$0.9 \mathrm{nA}$ ) this suggests that the lifetime scaling with laser 
spot size may not be significant until a certain beam intensity threshold is reached.

The lifetime as a function of active area is shown in Fig. 5. For identical laser spot size $(500 \mu \mathrm{m})$, limiting the photocathode active clearly enhances lifetime. For the small laser spot size lifetime worsens as the beam intensity is increased. For the larger active area the vacuum is sufficiently bad that the lifetime is poor at any beam intensity. Scaling the lifetime by the vacuum pressure for each run (see Fig. 6) shows the strong correlation between photocathode lifetime and the beam induced pressure rise due to photoemission at the photocathode edge.

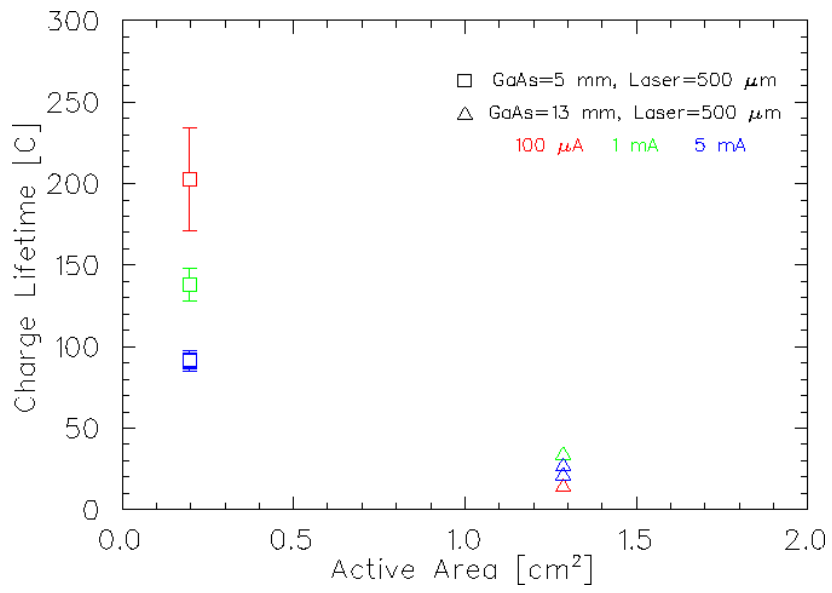

Figure 5: (Color) Photocathode lifetime versus active area with laser spot size fixed at $500 \mu \mathrm{m}$.

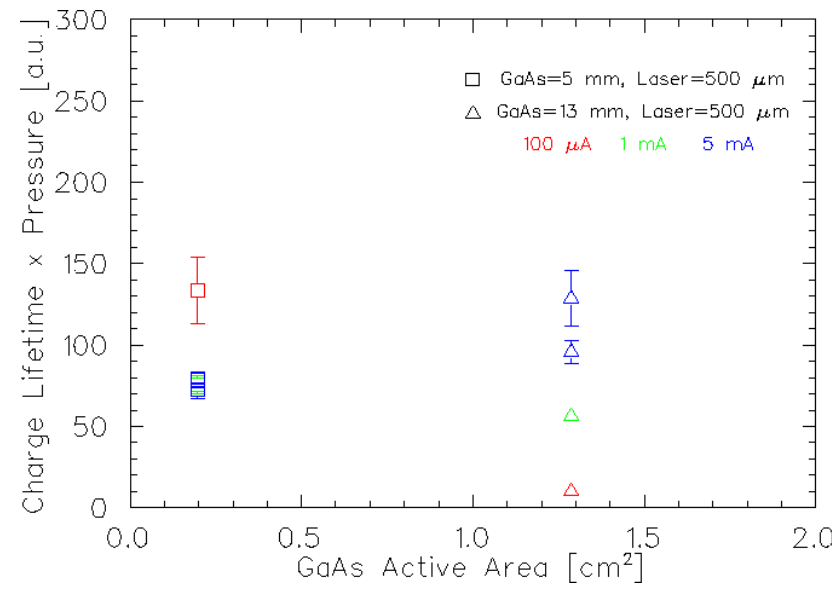

Figure 6: (Color) Product of photocathode lifetime and vacuum pressure versus active area with laser spot size fixed at $500 \mu \mathrm{m}$.

\section{CONCLUSIONS}

In this paper, a $100 \mathrm{kV}$ load locked gun and beam line are used to study GaAs photocathode lifetime as a function of beam current, photocathode active area, laser spot size and vacuum pressure of the gun and beam line. Lifetime measurements at $100 \mu \mathrm{A}$ help to benchmark the test apparatus against the CEBAF photoinjector, a machine recognized as having exceptionally good photocathode lifetime. Higher current operation at $1 \mathrm{~mA}$ and $5 \mathrm{~mA}$ are particularly interesting for high current FEL and ERL applications.

Two photocathode active areas were studied demonstrating the statement that eliminating inadvertent photoemission from the edge of the photocathode prolongs photocathode lifetime, particularly for systems with limited beam acceptance. In addition, lifetime was studied for two different laser spot sizes, to explore the possibility that one can expect longer gun operating lifetime by merely illuminating a larger spot on the photocathode. This idea stems from the notion that longer photocathode lifetime is obtained when ion damage is distributed over a large area. During these tests, we observed, under similar vacuum conditions, no improvement at $100 \mu \mathrm{A}$, marginal improvement at $1 \mathrm{~mA}$, and observed the anticipated scaling with laser spot size at $5 \mathrm{~mA}$. This suggests that another (current dependent) mechanism besides unwanted photoemission outside the acceptance of the gun plays a significant role in photocathode QE degradation. For example, the best lifetime was observed for $5 \mathrm{~mA}$ operation as opposed to $100 \mu \mathrm{A}$ operation (for large laser spot and small active area). This proceeding marks the beginning of a thorough study of lifetime effects for DC high voltage GaAs photoguns at beam currents suitable for new high current machines.

\section{REFERENCES}

[1] K. Aulenbacher et al., Operating Experience with the MAMI Polarized Electron Source, 1993 Proceedings of the Workshop on Photocathodes for Polarized Electron Sources for Accelerators, SLAC-R-432, Stanford, CA, 1994.

[2] C.K. Sinclair, Recent Advances in Polarized Electron Sources, Proceedings of the 1999 Particle Accelerator Conference, New York, NY, 1999.

[3] P.M. Rutt and A.R.Day, Active Area Definition of GaAs Photocathodes via Anodization, JLAB-TN-01-030, Newport News, VA, 2001.

[4] K. Aulenbacher, Overview of Polarized Electron Source Progress, Proceedings of the Ninth International Workshop on Polarized Sources and Targets, Nashville, IN, 2001.

[5] C. Hernandez-Garcia et al., A high average current DC GaAs photocathode gun for ERLs and FELs, Proceedings of the 2005 Particle Accelerator Conference, Knoxville, TN, 2005. 